\title{
RECONSTRUCTION OF FLOATING THUMB BY TRANSPLANTING THE FOURTH METATARSAL
}

\author{
AKIHITO TSUJINO, YOSHIYASU ITOH, KOICHIRO HAYASHI
}

From the University of Tsukuba and the Keiyu Orthopedic Hospital, Japan

We report the reconstruction of two cases of floating thumb by transplanting the distal two-thirds of the fourth metatarsal. Opponensplasty was performed after six months and resulted in satisfactory morphological and functional results. The metatarsal defect was filled by a full-thickness iliac bone graft including the apophysis. This prevented shortening of the fourth toe and formed a new metatarsophalangeal joint.

J Bone Joint Surg [Br] 1994; 76-B:551-4.

Received 22 November 1993; Accepted 5 January 1994

The management of floating thumb by either pollicisation of the index finger (Buck-Gramcko 1971) or thumb reconstruction (Tajima 1985) may involve difficult decisions. Parents are often very concerned about the number of digits and may not consent to pollicisation of the index finger although this gives good function. Thumb reconstruction, however, may lead to functional problems.

Reconstruction has been by carpometacarpal arthroplasty and opponensplasty. In one reported case a metatarsal was transferred (Yabe, Saito and Tsukimara 1973) and in another an iliac bone graft was used (Yamauchi et al 1979). Both provided good morphological and functional results, but there were problems of toe shortening after the use of a metatarsal graft and bone resorption with the iliac bone graft.

We have performed thumb reconstruction by transplantation of the fourth metatarsal and opponensplasty in two cases, also transplanting a full-thickness iliac-bone graft for repair of the metatarsal defect to prevent reduction of the fourth toe.

\section{OPERATIVE TECHNIQUE}

A dorsal sliding skin flap (Tajima 1985) is raised from the first web space, and the transverse metacarpal ligament

A. Tsujino, MD, Clinical Fellow

K. Hayashi, MD, PhD, Professor

Department of Orthopaedic Surgery, Institute of Clinical Medicine, University of Tsukuba, 1-1-1 Tennoudai, Tsukuba 305, Japan.

Y. Itoh, MD, PhD, Associate Director

Keiyu Orthopedic Hospital, 1741 Hanetsuku, Tatebayashi 374, Japan.

Correspondence should be sent to Dr A. Tsujino.

(C)1994 British Editorial Society of Bone and Joint Surgery 0301-620X/94/4810\$2.00 is incised to allow abduction of the floating thumb. The stump of the thumb metacarpal is freshened by bone shaving. The fascial covering of the first dorsal interosseous is incised longitudinally on the radial side, and the cartilaginous structures of the radial carpus, usually including the hypoplastic trapezium and scaphoid, are separated in a proximal direction. A shallow recess in the carpus is then prepared so that the transplanted bone can be stabilised.

The distal two-thirds of the fourth metatarsal is resected extraperiosteally and the metatarsal head is positioned in the prepared recess. Kirschner wires are inserted to maintain the position of the thumb, being passed into the carpus through the tip of the thumb and the transplanted bone. The area surrounding the metatarsal head is covered by the prepared cartilaginous flap and the fascia covering the first dorsal interosseous. The transplanted metatarsal is covered by the sliding skin flap, and the dorsal surface of the first web is closed by a fullthickness skin graft.

A full-thickness iliac bone graft, including the apophysis and the surrounding soft tissues, is then taken and inserted into the defect left by the metatarsal resection so that the iliac apophysis opposes the joint surface of the proximal phalanx. It is fixed in place by a Kirschner wire passed through from the tip of the fourth toe. The capsule of the metatarsophalangeal joint is sutured to the soft tissues of the apophysis of the iliac graft.

The thumb and the fourth toe remain fixed by the Kirschner wires for approximately six weeks, and then a splint is applied to maintain the abducted position of the thumb.

The muscles and tendons are hypoplastic or absent in many floating thumbs, and an opponensplasty is therefore performed about six months after the bone operation, when the surrounding tissues have stabilised. Abductor digiti quinti is transferred as first described by Huber (1921) and subsequently by Littler and Cooley (1963).

\section{CASE REPORTS}

Case 1. A six-month-old boy had left radial ray deficiency and a floating thumb. Only the head of the thumb metacarpal was present (Fig. 1a). Centralisation of the carpus (Delorme 1969) was the first stage, then at the age 


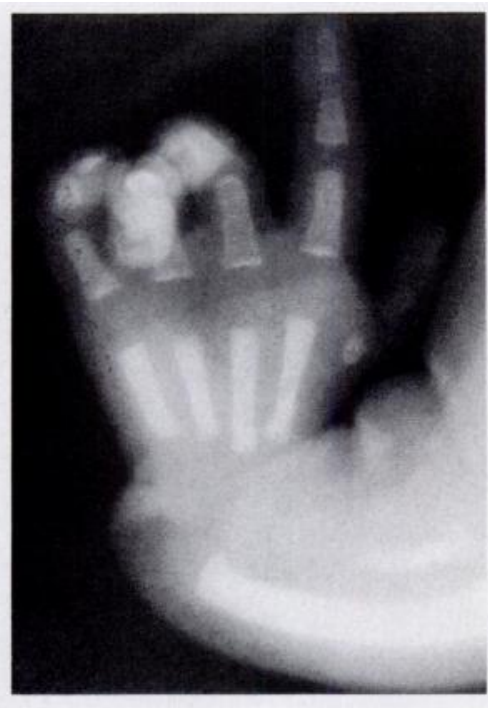

Fig. 1a

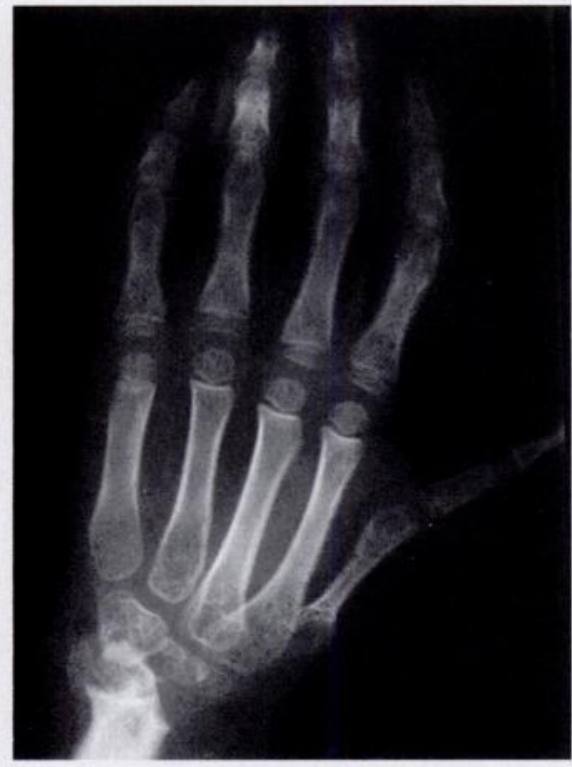

Fig. 1c

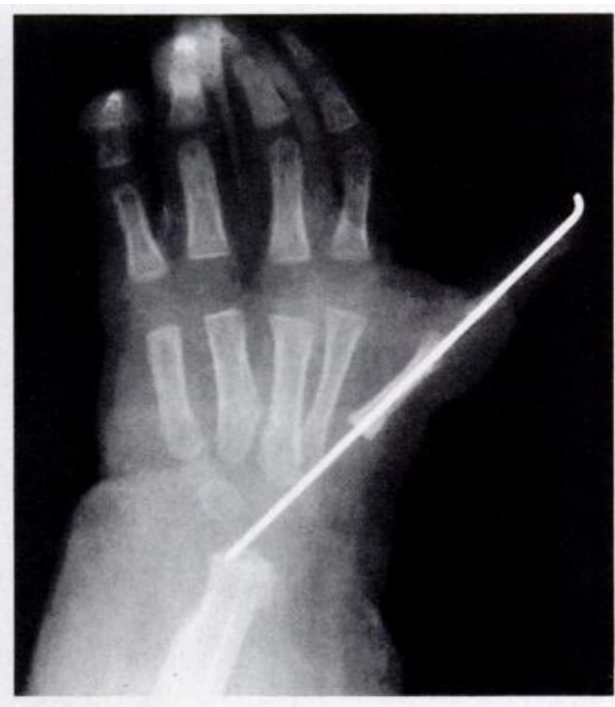

Fig. 1b

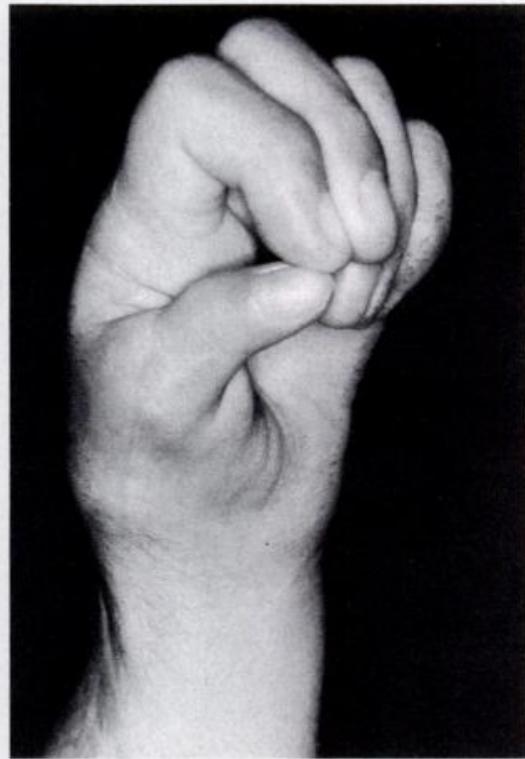

Fig. 1d
Case 1. Figure 1a - Radiograph of a sixmonth-old boy with left radial ray deficiency and a floating thumb. The thumb is smaller than the little finger and the metacarpal is represented only by the head of the bone. Figure $1 \mathrm{~b}-$ At 15 months, transplantation of the fourth metatarsal is held by Kirschner wire fixation. Figures $1 \mathrm{c}$ and $1 \mathrm{~d}-$ Nine years after transplantation, the transplanted metatarsal is alive and shows an epiphyseal line. The external appearance and opposition of the thumb are satisfactory. of 15 months, the fourth left metatarsal was transplanted (Fig. 1b) and simultaneously replaced in the foot by an iliac bone graft.

In this case, the first interosseous muscle was hypoplastic and the extensor, flexor and abductor of the thumb were absent. At the next stage, six months after bone transfer, thumb opposition was restored by transfer of the abductor digiti quinti, extension by transfer of the extensor indicis proprius and flexion by transfer of the fourth flexor digitorum superficialis tendon. Ulnar lengthening was required at the age of eight years and performed by external skeletal distraction. At the age of ten years, nine years after transplantation, the metatarsal graft remained viable with a visible epiphyseal line (Fig. 1c). Both the external appearance and the function of the thumb were satisfactory (Fig. 1d), and there was no shortening of the fourth toe.
Case 2. A boy aged 4 years 3 months presented with right radial ray deficiency and a floating thumb (Fig. 2a). Carpal centralisation had been performed at seven months. The thumb was triphalangeal, smaller than the little finger, and its metacarpal was represented only by the head of the bone. Thumb reconstruction was by transplantation of two-thirds of the fourth metatarsal (Fig. 2b) using an iliac bone graft to replace it (Fig. 3a). Opponensplasty was performed six months after the metatarsal transplant. Three years after transplantation, the metatarsal graft was viable, but showed partial epiphyseal arrest (Fig. 2c). The new carpometacarpal joint of the thumb was mobile, functioning with the transferred muscle, but the interphalangeal joint was stiff. The thumb was used for grasping and pinching. The metatarsophalangeal joint of the fourth toe, formed between the iliac bone graft and the proximal phalanx, appeared to be satisfactory. The 


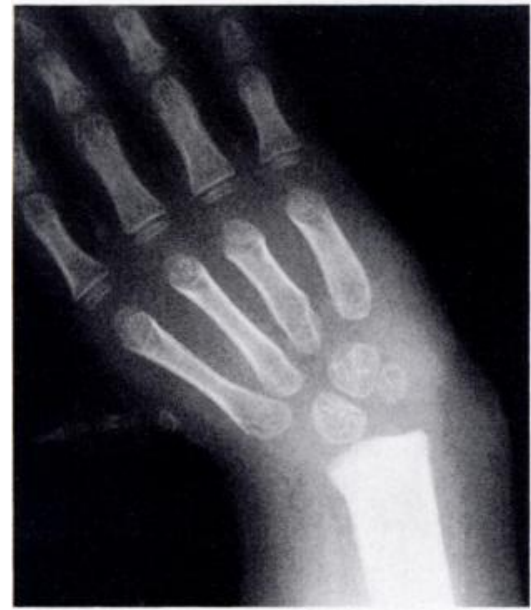

Fig. 2a

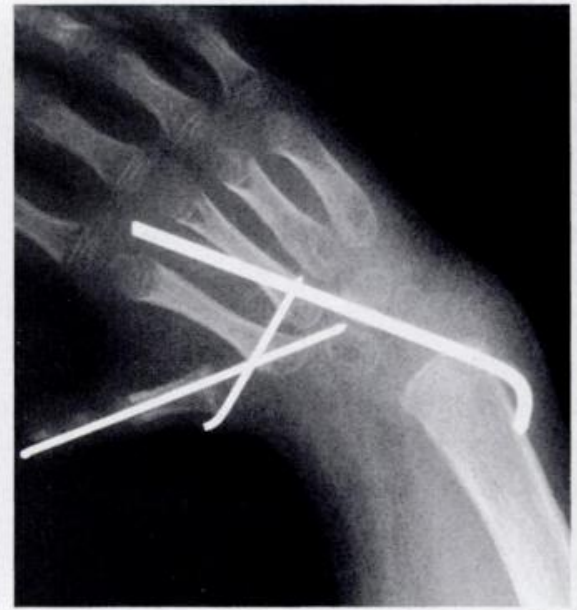

Fig. $2 b$

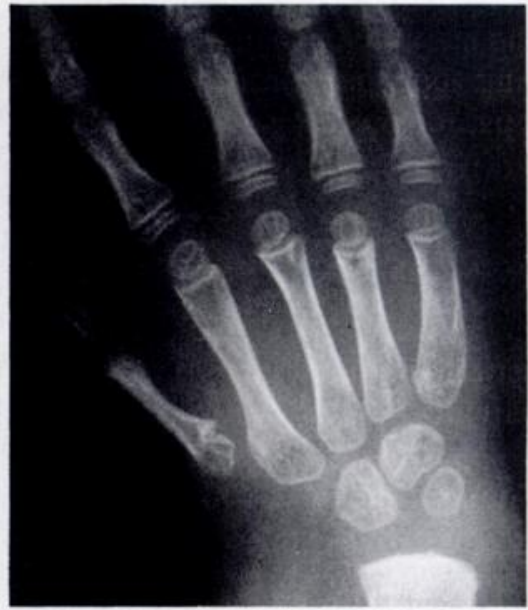

Fig. 2c

Case 2. Figure $2 \mathrm{a}$ - Radiograph of a boy aged 4 years 3 months with right radial ray deficiency and a floating thumb. Carpal centralisation had been previously performed. Figure $2 b-$ Transplantation of the fourth metatarsal with Kirschner wire fixation. Figure $2 c-$ Three years after transplantation, there is partial epiphyseal arrest.

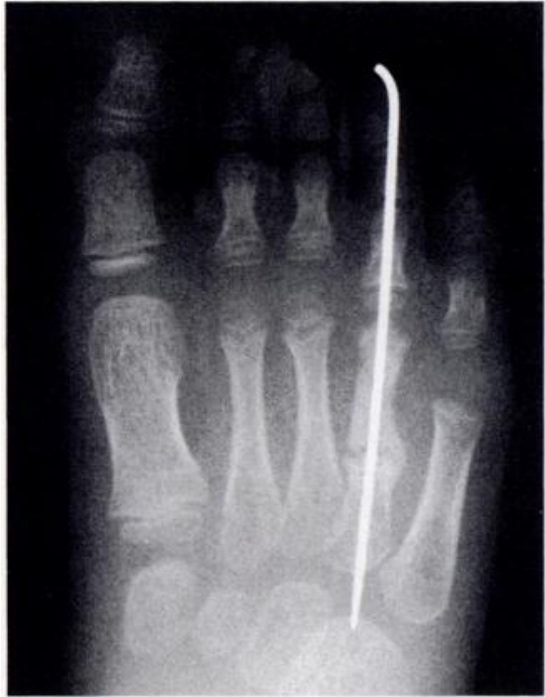

Fig. 3a

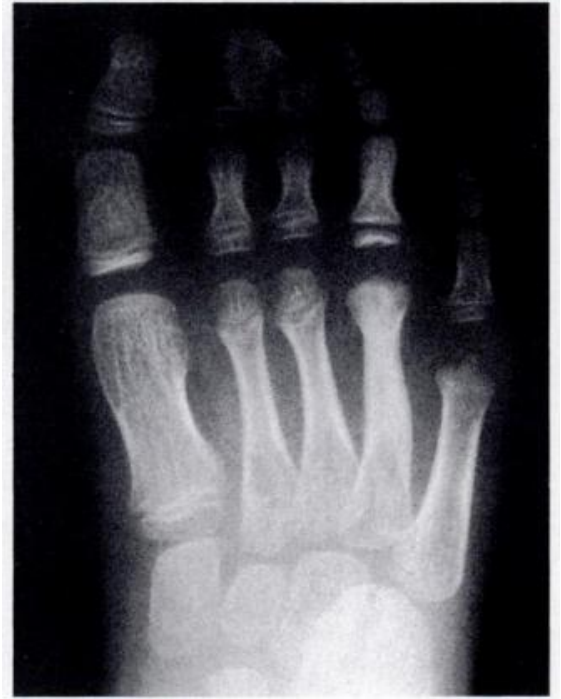

Fig. 3b
Case 2. Figure 3a - A full-thickness iliac bone graft, including the apophysis, was transferred to the metatarsal defect, with the apophysis opposite the articular surface of the proximal phalanx. Figure $3 b-$ Three years after transplantation the grafted metatarsal has a normal length and the metacarpophalangeal joint is satisfactory. iliac bone graft had grown and there was no reduction of the fourth toe (Fig. 3b).

\section{DISCUSSION}

Most floating thumbs are unilateral; bilateral cases are very rare. With one normal hand, general function is usually satisfactory and pollicisation of the index finger after excision of the floating thumb may not be indicated for purely functional reasons. Thumb restoration is more attractive to the patient and the parents because the number and shape of the digits are important considerations.

A mobile carpometacarpal joint is essential to the function of the thumb, and opponensplasty by transfer of the abductor digiti quinti is useless unless the potential first web space is increased by a mobile carpometacarpal joint and a sufficiently long metacarpal. An iliac bone graft transplanted to the thumb is likely to fuse with the carpal bones or the second metacarpal because cancellous bone surfaces are exposed. Fusion at the new carpometacarpal joint can be prevented by transplantation of the metatarsal with its intact articular surface. Yabe et al (1973) obtained a good result by transplantation of a metatarsal after excision of a failed, fused iliac bone graft. Yamauchi et al (1979), however, reported a case in which carpometacarpal mobility was achieved after the use of an iliac bone graft including its apophysis, although resorption of the bone graft took place later and iliac bone grafting had to be repeated (Tajima 1985). Such bone resorption does not appear to affect the metatarsal because it is a long bone and surrounded by periosteum.

Some epiphyseal arrest in the transplanted bone began early, but was not complete in case 1 after nine 
years and in case 2 after three years. This appears to show that not only the surface of the new carpometacarpal joint but also the cartilage of the epiphysis is viable and had contributed to longitudinal bone growth, even if this is not sufficient to maintain a normal amount of growth.

Shortening of the fourth toe is a recognised primary malformation of the foot for which operative treatment is sometimes required (Johnson 1972) because of the wishes of the parents and the patient. It is, therefore, important to avoid shortening of the toe after using the fourth metatarsal for thumb reconstruction. In our experience, the apophysis of an iliac crest graft remains viable, the new metatarsal grows longitudinally and the surface of the metatarsophalangeal joint is remodelled. Function is normal and the fourth toe is not shortened. Iliac bone transplanted to the foot was not resorbed, presumably because local blood flow is better there than after transplantation into a floating thumb.

We recommend treatment of floating thumb by transplantation of the fourth metatarsal and opponensplasty. Both appearance and function are satisfactory, the fourth toe is not shortened and a new metacarpophalangeal joint is formed by the full-thickness iliac bone graft.

No benefits in any form have been received or will be received from a commercial party related directly or indirectly to the subject of this article.

\section{REFERENCES}

Buck-Gramcko D. Pollicization of the index finger: method and results in aplasia and hypoplasia of the thumb. J Bone Joint Surg [Am] 1971; 53-A:1605-17.

Delorme TL. Treatment of congenital absence of the radius by transepiphyseal fixation. J Bone Joint Surg [Am] 1969; 51-A:117-29.

Huber E. Hilfsoperation bei Medianuslähmung. Deutsche Zeitschrift $f$. Chirurgie 1921; 162:271-5.

Johnson HA. Correction of a congenitally short fourth metatarsal. $\mathrm{Br} J$ Plast Surg 1972; 25:201.

Littler JW, Cooley SGE. Opposition of the thumb and its restoration by abductor digiti quinti transfer. J Bone Joint Surg [Am] 1963; 45A:1389-96.

Tajima T. Classification of thumb hypoplasia. Hand Clin 1985; 1:577-94.

Yabe Y, Saito M, Tsukimura T. Floating thumb. Seikei geka 1973; 24:1207-10. [In Japanese]

Yamauchi Y, Fujimaki A, Yanagihara Y, Yoshizaki K. Reconstruction of floating thumb: especially on the use of vascularized metatarsophalangeal joint grafting. Seikei geka 1979; 30:1645-8. [In Japanese] 